\title{
PERCEPCIÓN DE LOS SISTEMAS DE ENSEÑANZA-APRENDIZAJE Y EVALUACIÓN APLICADOS POR LOS PROFESORES EN LA REGIÓN JUNÍN
}

\section{PERCEPTION OF LEARNING SYSTEMS AND EVALUATION USED BY PROFFESORS IN JUNIN REGION}

\author{
Tello Yance, Jesús; Barrientos Gutiérrez, Pedro; Tello Matril, Lizett; Flores Muñoz, Celia Ruth
}

\section{RESUMEN}

El presente trabajo trata sobre la Percepción de los sistemas de enseñanza - aprendizaje y evaluación aplicados por los profesores en la Región Junín, el propósito de la investigación fue determinar la relación que existe entre la percepción de los sistemas de enseñanza aprendizaje y la evaluación aplicada por los docentes en la Región Junín. El tipo de investigación es el descriptivo y el diseño metodológico responde al descriptivo correlacional. La población estuvo constituida por los estudiantes del último grado de Educación Secundaria de Educación Básica Regular y la muestra estuvo representada por los 300 estudiantes, seleccionados por la fórmula de regla de tres simple. Para la recolección de datos se utilizó la técnica de la encuesta teniendo como instrumento el cuestionario. Entre las conclusiones tenemos: Con un nivel de significación $\alpha=0,05$ y para 2 grados de libertad se concluye que: El nivel de percepción de la evaluación del desempeño docente no está asociado al género de los estudiantes. Se demuestra estadísticamente que las mujeres no poseen mejor nivel de percepción de la evaluación del desempeño docente de la Región Junín, afirmación que se hace para un 95\% de nivel de confianza y 5\% de nivel de significación. Existe diferencia en las frecuencias de los niveles de percepción de la evaluación del desempeño docente, para un nivel de significación $\alpha=0,05$ y para 2 grados de libertad. Se demuestra mediante la estadística inferencial que existe un nivel Medio de percepción de la evaluación del desempeño docente en la Región Junín, conclusión que se hace para un 95\% de nivel de confianza y 5\% de nivel de significación y 2 Grados de libertad. Las alumnas $(2,87)$ presentan un puntaje mayor que los alumnos $(2,79)$ en la escala 1-4 de percepción del desempeño docente de la región Junín, lo que cualitativamente equivale a un nivel un nivel Medio en la escala en ambos grupos, esta afirmación se hace en base al contenido de la tabla No 4.8. Cualitativamente las alumnas y los alumnos tienen una percepción del desempeño docente en el nivel Medio en 7 dimensiones: Actividad en clase (2,95), Evaluación (2,94), Técnicas didácticas (2,86), Medios instruccionales (2,16), Participación de los estudiantes (2,97), Motivación $(2,80)$ y Técnicas para el establecimiento de conocimientos y habilidades $(2,87)$, mientras que en la dimensión Actitudes el nivel de percepción de las alumnas y alumnos es Alto, ya que tiene un puntaje de 3,10, afirmación que se hace en base a la tabla No 4.7.

Palabras clave: Sistema de enseñanza, aprendizaje

\section{ABSTRACT}

This paper deals with the perception of education systems - learning and assessment applied by teachers in Junín region, the purpose of the research was to determine the relationship between the perceptions of education systems applied learning and assessment for teachers in the region Junín. The research is descriptive and methodological descriptive correlational design responds to. The population consisted of students from the last grade of Secondary Education Basic Education Regular and the sample was represented by 300 students, selected by the formula of rule of three simple. The technique of the instrumentality of the survey questionnaire was used for data collection. Among the findings we are: With a level of $\alpha=0.05$ and 2 degrees of freedom significance concludes that: The level of perception of teacher evaluation is not associated with the gender of students. Statistically it demonstrates that women have better level of perception of performance evaluation teacher Junín region, a statement that is made for a 95\% confidence level and 5\% significance level. There is a difference in the frequencies of the levels 
of perception of teacher evaluation, to level $\alpha=0.05$ and 2 degrees of freedom significance. It is demonstrated by statistical inference that there is an average level of perception assessment teacher performance in Junín region, a conclusion is made for a 95\% confidence level and 5\% level significance and 2 degrees of freedom. The students (2.87) have a higher score than the students (2.79) on 1-4 scale perception teaching performance of the Junin region, a level qualitatively equivalent to an average level on the scale both groups, this statement is based on the contents of the table No 4.8. Qualitatively the pupils and students have a perception of teaching performance in the Middle level 7 dimensions: Class Activity (2.95) Rating (2.94), teaching techniques (2.86), instructional media (2.16) Student participation (2.97), Motivation (2.80) and Techniques for the establishment of knowledge and skills (2.87), while the dimension poses the level of perception of the pupils and students is high, as it has a score of 3.10 , the statement is made based table № 4.7

Keywords: Education system learning

\section{INTRODUCCIÓN}

La evaluación es un proceso que forma parte de la enseñanza y del aprendizaje que permite al docente observar, recoger, describir, analizar y explicar información importante acerca de: posibilidades, necesidades, logros del estudiante con la finalidad de reflexionar, valorar y tomar decisiones oportunas para mejorar la enseñanza y el aprendizaje (MED, 2008). Sin embargo, en la práctica pedagógica no se concreta esta concepción de la evaluación, más bien generamos miedo y rechazo en los estudiantes.

Ello reviste de singular importancia en la realización del presente trabajo de investigación: Percepción de los sistemas de enseñanza - aprendizaje y evaluación aplicados por los profesores en la región Junín; cuyo propósito fundamental es establecer la relación que existe entre la percepción de los sistemas de enseñanza aprendizaje y evaluación aplicados por los profesores en la región Junín, con la finalidad de obtener información suficiente para tomardecisiones que permita mejorar en las instituciones educativas de la región de Junín.

El contenido del presente trabajo de investigación está estructurado en cinco capítulos: el primero está referido al planteamiento del problema de investigación, en el que se presenta el título de la investigación, descripción del problema, formulación del problema y los objetivos de la investigación; el segundo trata acerca del marco teórico, en la que se desarrollan las bases teóricas y las hipótesis del estudio; el tercero relativo a los materiales y métodos, donde se presenta el tipo y nivel de investigación, métodos de la investigación, población y muestra de estudio, así como las técnicas e instrumentos de recopilación de datos; el cuarto está referido a los resultados de la investigación, en la que presentamos los resultados obtenidos mediante tablas estadísticos; y el quinto se trata de la discusión de los resultados. Finalmente presentamos las conclusiones, sugerencias y los anexos.

En la realización del presente estudio se ha tenido dificultades en cuanto a la recolección de la información debido a la distancia de las muestras seleccionadas y limitaciones de carácter metodológico, la cual fue superada con las atingencias en la elaboración de los instrumentos de recolección de la información.

Para terminar, expresamos nuestra imperecedera y sincera gratitud a todas aquellas personas que con su valiosa y desinteresada colaboración contribuyeron a la exitosa realización del presente trabajo de investigación.

\section{MATERIAL Y MÉTODOS}

\section{Tipo de investigación}

El presente trabajo de investigación responde a una tipología de investigación descriptiva, que consistió en describir situaciones o fenómenos referidos, en este caso, del sistema de enseñanza-aprendizaje y la evaluación aplicados por los docentes en la Región Junín (Barrientos, 2005, p. 53).

\section{Diseño metodológico de la investigación}

De acuerdo a los objetivos y la hipótesis planteada, el estudio se ha guiado a través del diseño descriptivo corre- 
lacional de investigación, lo cual permitido establecer la relación que existe entre las variables del sistema de enseñanza-aprendizaje y la evaluación aplicados por los docentes en la Región Junín; cuyo esquema es el siguiente:

\section{M: Muestra}

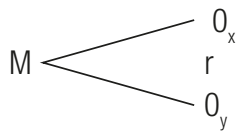

0: Información

$X:$ dato de sistema de $\mathrm{E}-\mathrm{A}$

$Y$ : dato de sistema de evaluación

$r$ : Representa la relación probable entre las variables.

\section{Método de estudio.}

El método es un elemento necesario en la ciencia; ya que sin él no sería fácil demostrar si un argumento es válido. El presente trabajo de investigación se ha guiado por el método No experimental y de manera específica por el método hipotético deductivo, Según SIERRA (2002, p.30), se entiende por método, como procedimiento, está constituido por las etapas generales de actuación que forma su contenido y por las técnicas y procedimientos concretos, operativos, para realizar en un caso determinado las fases generales de actuación en cuestión.

\section{POBLACIÓN Y MUESTRA}

\section{- Población total}

Todos los estudiantes de la Región Junín

\section{- Población accesible}

10\% de los estudiantes de la Región Junín

\section{- Muestra}

300 estudiantes seleccionados

\section{- Fórmula de la muestra}

Por la naturaleza de la investigación se aplicará la fórmula de regla de tres simple.

\section{Técnicas e instrumentos de recolección de datos}

En la presente investigación, la información se ha obtenido en dos fases, la primera consistió en el trabajo de campo se efectúa el encuentro entre el investigador y el fenómeno estudiado, la segunda fase es el trabajo en gabinete consiste en el análisis de los datos mediante el uso de la estadística.

Las técnicas y los instrumentos que se utilizó en la fase de trabajo de campo fue la encuesta, teniendo como instrumento el cuestionario que fueron aplicados a los estudiantes de los últimos grados de Educación Secundaria.

\section{Técnicas de procesamiento y análisis de datos}

Los datos obtenidos a través de la aplicación de las técnicas y los instrumentos de recopilación de datos fueron organizados y procesados mediante tablas y gráficos estadísticos de tendencia central para su correspondiente análisis a través de la estadística descriptiva e inferencial.

\section{PRESENTACIÓN, ANALISIS E INTERPRETACIÓN DE LOS RESULTADOS}

En el presente trabajo de investigación se estudia la percepción del sistema de enseñanza-aprendizaje y evaluación aplicados por los profesores de la Región Junín, para lo cual se tomó una muestra de 398 estudiantes de educación secundaria, de los cuales 272 son mujeres y 126 son alumnos varones. En este capítulo se describen y explican los hallazgos en relación a las mediciones realizadas a los alumnos en la investigación, con su correspondiente análisis estadístico e interpretación.

Análisis de los resultados de la percepción del sistema de enseñanza-aprendizaje y evaluación.

A continuación se presenta los resultados de la percepción de la variable Enseñanza-aprendizaje y evaluación de los alumnos de educación secundaria de la Región Junín.

\section{Análisis del los resultados de la percepción de las alumnas}

La tabla № 4.1 muestra los resultados de la percepción de las alumnas sobre la enseñanza-aprendizaje y evaluación que aplican los profesores en la región Junín.

Tabla № 4.1: Resultados de la percepción de las alumnas sobre la enseñanza-aprendizaje y evaluación de sus profesores

\begin{tabular}{|l|c|c|c|c|c|c|c|c|}
\hline \multirow{2}{*}{ Dimensiones } & \multicolumn{2}{|c|}{ Siempre } & \multicolumn{2}{c|}{$\begin{array}{c}\text { Frecuente } \\
\text { mente }\end{array}$} & \multicolumn{2}{c|}{ A veces } & \multicolumn{2}{c|}{ Nunca } \\
\cline { 2 - 9 } & fi & $\%$ & fi & $\%$ & fi & $\%$ & fi & $\%$ \\
\hline 1. Actividad en clase & 96 & 35,44 & 95 & 34,90 & 65 & 24,07 & 15 & 5,60 \\
\hline 2. Evaluación & 99 & 36,49 & 88 & 32,26 & 60 & 21,92 & 25 & 9,34 \\
\hline $\begin{array}{l}\text { 3. Técnicas didácti- } \\
\text { cas }\end{array}$ & 92 & 33,82 & 86 & 31,68 & 68 & 25,03 & 26 & 9,46 \\
\hline
\end{tabular}




\begin{tabular}{|l|c|c|c|c|c|c|c|c|}
\hline $\begin{array}{c}\text { 4.Medios instruc- } \\
\text { cionales }\end{array}$ & 38 & 13,73 & 49 & 17,72 & 102 & 37,47 & 83 & 31,08 \\
\hline $\begin{array}{c}\text { 5.Participación de } \\
\text { estudiantes }\end{array}$ & 95 & 34,87 & 95 & 34,99 & 73 & 26,65 & 10 & 3,49 \\
\hline 6. Motivación & 83 & 30,51 & 82 & 30,15 & 84 & 30,81 & 23 & 8,53 \\
\hline 7. Actitudes & 119 & 43,75 & 97 & 35,59 & 48 & 17,79 & 8 & 2,87 \\
\hline $\begin{array}{c}\text { 8. Conocimiento y } \\
\text { habilidades }\end{array}$ & 81 & 29,85 & 101 & 36,99 & 73 & 26,69 & 18 & 6,47 \\
\hline
\end{tabular}

Fuente: Encuesta realizada por Jesús Tello y Pedro Barrientos (30-10-2012).

Se observa que el 35,44\% de las alumnas señalan que Siempre sus profesores desarrollan la actividad de la clase explicando con diferentes niveles de complejidad, resolviendo dudas planteadas, con voz clara, de manera entendible, con mucha fluidez, de acuerdo a la capacidad de asimilación de los alumnos, manteniendo contacto visual, con una cantidad suficiente de material didáctico, sin faltas ortográficas, con letra clara y legible. El 34,90\% de las alumnas señala que sus profesores lo realizan frecuentemente, el 24,07\% manifiesta que los profesores lo hacen a veces y el 5,60\% indica que sus profesores nunca lo hacen.

Con respecto a la evaluación, se observa que la mayoría $(36,49 \%)$ de las alumnas manifiestan que Siempre sus profesores realizan una evaluación escrita, oral, evalúan mediante ensayos, prácticas, evalúan la asistencia y puntualidad, los reportes de investigación y la participación en clase. También señalan que Siempre los profesores han evaluado lo expuesto en clase, los objetivos del sílabo, las lecturas complementarias y que la frecuencia fue semanal, mensual y semestral. El 32,26\% de los alumnos señalan que fue frecuentemente, el $21,92 \%$ de las alumnas indicaron que fue A veces y sólo el 9,34\% señaló que Nunca los profesores realizaron la correspondiente evaluación.

La mayoría $(33,82 \%)$ de las alumnas señalan que Siempre los profesores de la Región Junín han expuesto de manera verbal, han explicado y ejecutado directamente el procedimiento que van enseñar, han proporcionado una guía, han invitado a un grupo de personas competentes en los temas expuestos, han reunido a los alumnos en pequeños grupos y en parejas para discutir un tema, han invitado a expertos y generado espacios para opinar con libertad. El 31,68\% de las alumnas manifiestan que los profesores lo han hecho con frecuencia.

En relación al uso de los medios instruccionales, la mayoría $(37,47 \%)$ de las alumnas señalan que los profesores han utilizado A veces grabadora, radios, pizarrón, rotafolios, láminas y carteles, material impreso, periódico mural, franelógrafos, fotografías, retroproyector, proyector multimedia, proyector de cine, equipos de video, computadora o televisión. El 31,08\% de las alumnas señalan que Nunca han utilizad los medios instruccionales, el $17,72 \%$ de las alumnas señalan que han utilizado los medios instruccionales Frecuentemente y sólo el 13,73\% de las alumnas indican que han utilizado Siempre los medios instruccionales en las clases.

La mayoría $(34,99 \%)$ de las alumnas encuestadas señalan que Frecuentemente los profesores han organizado a los alumnos para las exposiciones en grupo, han propiciado la aclaración de dudas, han realizado investigaciones teórico-prácticas, han dado ejemplos en el material asignado, y han presentado casos para el correspondiente análisis. El 34,87\% de las alumnas señalan que los profesores han propiciado la participación de los estudiantes Siempre, el 26,65\% de las alumnas han manifestado que ha sido A veces y el 3,49\% de las alumnas encuestadas han señalado que los profesores Nunca han propiciado la participación de los alumnos.

La mayoría $(30,81 \%)$ de las alumnas manifiestan que los profesores frecuentemente han motivado a los alumnos en las clases, es decir se han comunicado individualmente con los alumnos, han señalado objetivamente los aciertos y errores de los alumnos, han planteado nuevos problemas y preguntas motivadoras, han estimulado los alumnos. El 30,51\% de las alumnas encuestados han indicado que Siempre los profesores han motivado a los alumnos, el $30,15 \%$ de las alumnas indican que Frecuentemente los profesores los han motivado y 8,53\% señala que Nunca los profesores los han motivado.

La mayoría $(43,75 \%)$ de la alumnas manifiestan que Siempre los profesores han demostrado actitudes en la Enseñanza-aprendizaje, es decir han demostrado dominio, responsabilidad, habilidad verbal, han ejemplificado con casos reales, han demostrado humor, accesibilidad al diálogo, y la crítica, imparcialidad, interés por su trabajo, asistencia a clases y puntualidad. El 35,59\% de las alumnas señalan que frecuentemente los profesores han demostrado actitudes favorables en su desempeño docente, el $17,79 \%$ indican que fue $A$ veces y el $2,87 \%$ de las alumnas encuestadas señalan que Nunca los profesores han demostrado actitudes favorables en su desempeño. 
Con respecto al desarrollo de las técnicas para el establecimiento de conocimientos y habilidades de los alumnos, la mayoría $(36,99 \%)$ de las alumnas señalan que Frecuentemente los profesores han recompensado el trabajo de los alumnos, han propiciado la transferencia a nuevas situaciones, han ejemplificado las actividades, han guiado al perfeccionamiento de habilidades. El 29,85\% de las alumna indican que los profesores Siempre han propiciado el desarrollo de conocimiento y habilidades de los alumnos, el 30,81\% indican que fue A veces y el 8,53\% manifiestan que Nunca los profesores han desarrolla las técnicas para el establecimiento de conocimiento y habilidades en los alumnos.

Tabla No 4.2: Resultados total de la percepción de las alumnas sobre la enseñanza-aprendizaje y evaluación

\begin{tabular}{|c|c|c|}
\hline Respuesta & $\mathrm{fi}$ & $\%$ \\
\hline Siempre & 88 & 32,35 \\
\hline Frecuentemente & 87 & 31,99 \\
\hline A veces & 72 & 26,47 \\
\hline Nunca & 25 & 9,19 \\
\hline Total & 272 & 100,00 \\
\hline
\end{tabular}

Fuente: Encuesta realizada por Jesús Tello y Pedro Barrientos (30-10-2012).

Se observa, en la tabla № 4.2, que la mayoría $(32,35 \%)$ de las alumnas opinan que los docentes Siempre han realizado las actividades de clase que han planificado, han evaluado y han utilizado las técnicas didácticas pertinentes, han utilizado los medios instruccionales adecuados, han propiciado la participación de los estudiantes, han motivado frecuentemente y han fomentado el desarrollo de actitudes en los alumnos de educación secundaria. El 31,99\% han realizado las actividades de la enseñanza-aprendizaje y evaluación de manera Frecuente, el 26,47\% de las alumnas indicaron que $A$ veces han realizado las acciones de la enseñanza-aprendizaje y el 9,19\% de las alumnas han indicado que Nunca ha realizado las actividades en referencia.

En el gráfico № 4.1 se observa que 175 alumnas perciben que Siempre o Frecuentemente los profesores de la Región Junín realizan la enseñanza-aprendizaje y evaluación, de acuerdo a las dimensiones e indicadores que se muestran en el anexo.

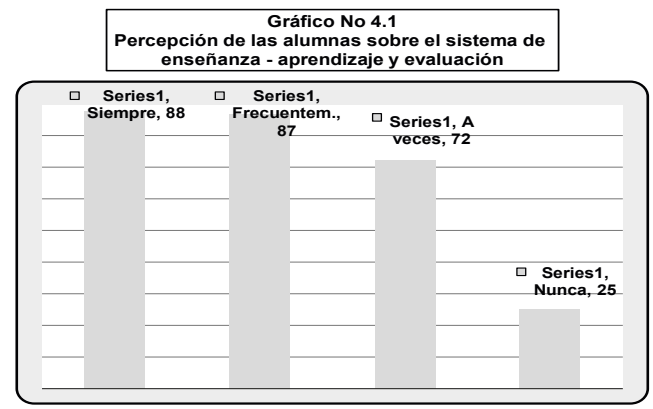

\section{Análisis del los resultados de la percepción de los alumnos}

Se presenta, en la tabla № 4.3 , los resultados de la percepción de los alumnos sobre el proceso de enseñanza-aprendizaje y evaluación que aplican los profesores en la región Junín, que han evaluados por los alumnos de la muestra de investigación.

\section{Tabla № 4.3}

Resultados de la percepción de los alumnos sobre la enseñanza-aprendizaje y evaluación de sus profesores

\begin{tabular}{|l|c|c|c|c|c|c|c|c|}
\hline \multirow{2}{*}{ Dimensiones } & \multicolumn{2}{|c|}{ Siempre } & \multicolumn{2}{|c|}{$\begin{array}{c}\text { Frecuen- } \\
\text { te-mente }\end{array}$} & \multicolumn{2}{|c|}{ A veces } & \multicolumn{2}{|c|}{ Nunca } \\
\cline { 2 - 10 } & $\mathrm{fi}$ & $\%$ & $\mathrm{fi}$ & $\%$ & $\mathrm{fi}$ & $\%$ & $\mathrm{fi}$ & $\%$ \\
\hline 1. Actividad en clase & 38 & 30,40 & 42 & 33,21 & 40 & 32,05 & 5 & 4,33 \\
\hline 2. Evaluación & 44 & 35,13 & 39 & 30,59 & 33 & 26,48 & 10 & 7,80 \\
\hline $\begin{array}{l}\text { 3. Técnicas didácti- } \\
\text { cas }\end{array}$ & 36 & 28,72 & 43 & 33,77 & 36 & 28,28 & 12 & 9,24 \\
\hline $\begin{array}{l}\text { 4. Medios instruc- } \\
\text { cionales }\end{array}$ & 18 & 14,42 & 24 & 18,65 & 44 & 34,63 & 41 & 32,30 \\
\hline $\begin{array}{l}\text { 5. Participación de } \\
\text { estudiantes }\end{array}$ & 38 & 29,76 & 49 & 38,89 & 33 & 26,06 & 7 & 5,29 \\
\hline 6. Motivación & 32 & 25,71 & 41 & 32,86 & 42 & 33,02 & 11 & 8,41 \\
\hline 7. Actitudes & 42 & 33,55 & 46 & 36,86 & 31 & 24,51 & 6 & 5,07 \\
\hline $\begin{array}{l}\text { 8. Conocimiento y } \\
\text { habilidades }\end{array}$ & 33 & 25,95 & 49 & 38,57 & 36 & 28,41 & 9 & 7,06 \\
\hline
\end{tabular}

Fuente: Encuesta realizada por Jesús Tello y Pedro Barrientos (30-10-2012).

Se observa que la mayoría $(35,44 \%)$ de los alumnos manifiestan que Frecuentemente sus profesores desarrollan la actividad de la clase explicando con diferentes niveles de complejidad, resolviendo dudas planteadas, con voz clara, de manera entendible, con mucha fluidez, de acuerdo a la capacidad de asimilación de los alumnos, manteniendo contacto visual, con una cantidad suficiente de material didáctico, sin faltas ortográficas, con letra clara y legible. El 32,05\% de los alumnos 
manifiestan que sus profesores lo realizan A veces, el $30,40 \%$ de los alumnos señalan que los profesores Siempre realizan las actividades en clase y el $4,33 \%$ indica que los profesores Nunca lo hacen.

En relación a la dimensión Evaluación, se observa que la mayoría $(35,13 \%)$ de los alumnos manifiestan que Siempre sus profesores realizan una evaluación escrita, oral, evalúan mediante ensayos, prácticas, evalúan la asistencia y puntualidad, los reportes de investigación y la participación en clase, han evaluado lo expuesto en clase, los objetivos del sílabo, las lecturas complementarias y que la frecuencia fue semanal, mensual y semestral. El 30,59\% de los alumnos señalan que fue frecuentemente, el $26,48 \%$ de los alumnos indicaron que fue A veces y sólo el 7,80\% señaló que Nunca los profesores realizaron la respectiva evaluación.

La mayoría $(33,77 \%)$ de los alumnos señalan que Frecuentemente los profesores de la Región Junín han utilizado técnicas didácticas, es decir han expuesto de manera verbal, han explicado y ejecutado directamente el procedimiento que van enseñar, han proporcionado una guía, han invitado a un grupo de personas competentes en los temas expuestos, han reunido a los alumnos en pequeños grupos y en parejas para discutir un tema, han invitado a expertos y generado espacios para opinar con libertad. El 28,72\% de los alumnos manifiestan que los profesores lo han hecho Siempre, el $28,28 \%$ de los alumnos manifiestan que los profesores A veces han utilizado técnicas didácticas apropiadas, y el 9,24\% de los alumnos señalan que Nunca los profesores han utilizado técnicas didácticas pertinentes.

Con respecto a la utilización medios instruccionales, la mayoría $(34,63 \%)$ de los alumnos señalan que los profesores han utilizado A veces grabadora, radios, pizarrón, rotafolios, láminas y carteles, material impreso, periódico mural, franelógrafos, fotografías, retroproyector, proyector multimedia, proyector de cine, equipos de video, computadora o televisión. El 32,30\% de los alumnos señalan que Nunca han utilizad los medios instruccionales, el 18,65\% de los alumnos señalan que han utilizado los medios instruccionales Frecuentemente y sólo el 14,42\% de los alumnos indican que han utilizado Siempre los medios instruccionales en las clases.

La mayoría $(38,89 \%)$ de los alumnos encuestados señalan que Frecuentemente los profesores han orga- nizado a los alumnos para las exposiciones en grupo, han propiciado la aclaración de dudas, han realizado investigaciones teórico-prácticas, han dado ejemplos en el material asignado, y han presentado casos para el correspondiente análisis. El 29,76\% de los alumnos señalan que los profesores han propiciado la participación de los estudiantes Siempre, el 26,06\% de los alumnos han manifestado que ha sido A veces y el 5,29\% de los alumnos encuestados han señalado que los profesores Nunca han propiciado la participación de los alumnos.

La mayoría (33,02\%) de los alumnos manifiestan que los profesores frecuentemente han motivado a los alumnos en las clases, es decir se han comunicado individualmente con los alumnos, han señalado objetivamente los aciertos y errores de los alumnos, han planteado nuevos problemas y preguntas motivadoras, han estimulado los alumnos. El 32,86\% de los alumnos encuestados han indicado que Frecuentemente los profesores han motivado a los alumnos, el $25,71 \%$ de los alumnos indican que Siempre los profesores los han motivado y $8,41 \%$ señala que Nunca los profesores los han motivado.

La mayoría (36,86\%) de los alumnos manifiestan que Frecuentemente los profesores han demostrado actitudes en la Enseñanza-aprendizaje, es decir han demostrado dominio, responsabilidad, habilidad verbal, han ejemplificado con casos reales, han demostrado humor, accesibilidad al diálogo, y la crítica, imparcialidad, interés por su trabajo, asistencia a clases y puntualidad. El 33,55\% de los alumnos señalan que Siempre los profesores han demostrado actitudes favorables en su desempeño docente, el 24,51\% indican que fue A veces y el $5,07 \%$ de los alumnos encuestados señalan que Nunca los profesores han demostrado actitudes favorables en su desempeño.

Con respecto al desarrollo de las técnicas para el establecimiento de conocimientos y habilidades de los alumnos, la mayoría $(38,57 \%)$ de los alumnos señalan que Frecuentemente los profesores han recompensado el trabajo de los alumnos, han propiciado la transferencia a nuevas situaciones, han ejemplificado las actividades, han guiado al perfeccionamiento de habilidades. El $28,41 \%$ de los alumnos indican que los profesores A veces han propiciado el desarrollo de conocimiento y habilidades de los alumnos, el 25,95\% indican que fue Siempre y el 7,06\% manifiestan que Nunca los profe- 
sores han desarrollado las técnicas para el establecimiento de conocimiento y habilidades en los alumnos.

Tabla № 4.4: Resultados total de la percepción de los alumnossobre laenseñanza-aprendizajey evaluación

\begin{tabular}{|l|c|c|}
\hline Respuesta & fi & $\%$ \\
\hline Siempre & 35 & 27,78 \\
\hline Frecuentemente & 41 & 32,54 \\
\hline A veces & 37 & 29,37 \\
\hline Nunca & 13 & 10,32 \\
\hline \multicolumn{1}{|c|}{ Total } & 126 & 100,00 \\
\hline
\end{tabular}

Fuente: Encuesta realizada por Jesús Tello y Pedro Barrientos (30-10-2012).

En la tabla № 4.4, se observa que la mayoría $(32,54 \%)$ de los alumnos opinan que los docentes Frecuentemente han realizado las actividades de clase que han planificado, han evaluado, han utilizado las técnicas didácticas pertinentes, han utilizado los medios instruccionales adecuados, han propiciado la participación de los estudiantes, han motivado frecuentemente y han fomentado el desarrollo de actitudes en los alumnos de educación secundaria. El 29,37\% han realizado las actividades de la enseñanza-aprendizaje y evaluación A veces, el $27,78 \%$ de los alumnos indicaron que Siempre han realizado las acciones de la enseñanza-aprendizaje y el 10,32\% de los alumnos han indicado que Nunca ha realizado las actividades en referencia.

En el gráfico № 4.2 se observa que 76 alumnos perciben que Siempre o Frecuentemente los profesores de la Región Junín realizan la enseñanza-aprendizaje y evaluación, de acuerdo a las dimensiones e indicadores que se muestran en el anexo del presente informe, hay 37 alumnos que tienen una percepción $A$ veces y 13 alumnos que señalan Nunca.

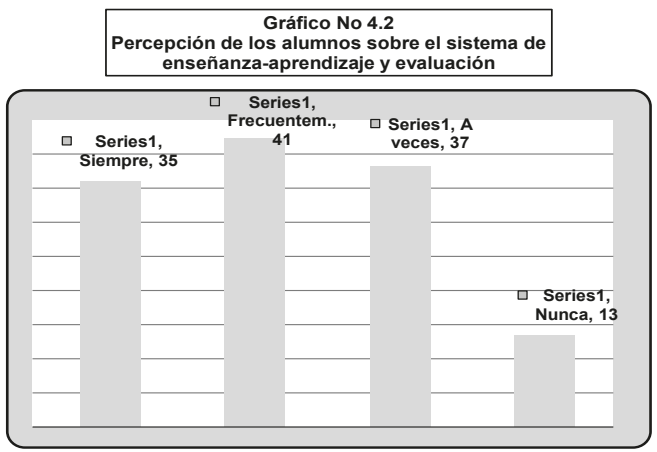

\section{Comparación de la percepción de las alumnas y alumnos}

Se observa, en la tabla № 4.5, que la mayoría $(32,35 \%)$ de las alumnas perciben que Siempre los profesores han desarrollado un sistema de enseñanza-aprendizaje y evaluación durante las clases, mientras que la mayoría $(32,54 \%)$ de los alumnos indican que la percepción es Frecuentemente sobre el desarrollo del sistema de enseñanza-aprendizaje y evaluación. En relación a la respuesta $A$ veces, se observa que hay un mayor porcentaje de percepción de los alumnos $(29,37 \%)$ en comparación a la respuesta de las alumnas $(26,47 \%)$. Con respecto a la respuesta Nunca, se aprecia que los alumnos presentan un mayor porcentaje de percepción. En suma, se puede concluir que las alumnas tienen una mejor percepción de la evaluación del desempeño docente, en relación a la percepción de las alumnas.

\section{Tabla № 4.5: Comparación de la percepción de las alumnas y los alumnos sobre la enseñanza- aprendizaje y evaluación}

\begin{tabular}{|l|c|c|c|c|}
\hline \multirow{2}{*}{ Respuesta } & \multicolumn{2}{c|}{ Mujeres } & \multicolumn{2}{c|}{ Varones } \\
\cline { 2 - 5 } & $\mathrm{fi}$ & $\%$ & $\mathrm{fi}$ & $\%$ \\
\hline Siempre & 88 & 32,35 & 35 & 27,78 \\
\hline Frecuentemente & 87 & 31,99 & 41 & 32,54 \\
\hline A veces & 72 & 26,47 & 37 & 29,37 \\
\hline Nunca & 25 & 9,19 & 13 & 10,32 \\
\hline \multicolumn{1}{|c|}{ Total } & 272 & 100,00 & 126 & 100,00 \\
\hline
\end{tabular}

Fuente: Encuesta realizada por Jesús Tello y Pedro Barrientos (30-10-2012).

En el gráfico № 4.3 se observa de manera visual las diferencias en la percepción de las alumnas y los alumnos, concluyendo que las alumnas presentan una mayor percepción del sistema de enseñanza-aprendizaje y evaluación que realizan los docentes observados.

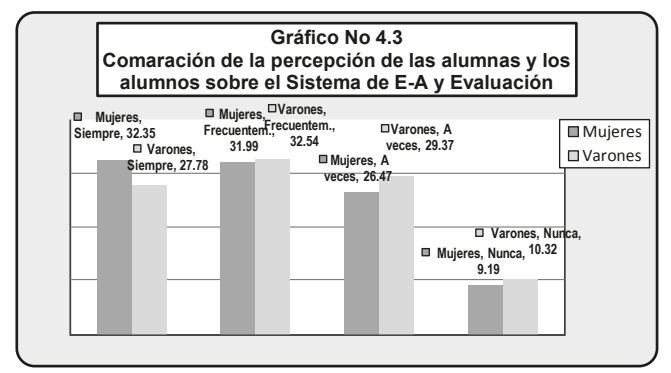


Se observa, en la tabla № 4.6, que cualitativamente las alumnas presentan una mejor percepción del desempeño docente en las dimensiones: Actividades en clase $(3,00)$, Participación de los estudiantes $(3,01)$ y las Actitudes $(3,20)$, ya que presentan un nivel Alto, mientras que los alumnos tienen una percepción menor, ya que presentan un nivel Medio. En las demás dimensiones: Evaluación, Técnicas didácticas, Medios instruccionales, Motivación y Técnicas para el establecimiento de conocimientos y habilidades, el nivel en ambos grupos es similar, ya que presentan un nivel Medio.

En relación a las ocho dimensiones, es decir en relación a la variable: Desempeño docente, se observa que ambos grupos presentan un nivel Medio de la percepción del sistema de enseñanza-aprendizaje y evaluación aplicados por los profesores de la Región Junín, aunque las alumnas $(2,87)$ han alcanzado un mayor puntaje en la escala de 1-4, en comparación a los alumnos, que tienen un puntaje de 2,79 en la escala 1-4.

Tabla № 4.6: Comparación de la percepción de las alumnas y los alumnos sobre la enseñanza-aprendizaje y evaluación por dimensiones

\begin{tabular}{|l|c|c|c|c|c|c|}
\hline \multirow{2}{*}{ Dimensiones } & \multicolumn{3}{|c|}{ Mujeres } & \multicolumn{3}{c|}{ Varones } \\
\cline { 2 - 7 } & $\begin{array}{c}\text { Ptje } \\
(272)\end{array}$ & $\begin{array}{c}\text { Escala } \\
(1-4)\end{array}$ & Nivel & $\begin{array}{c}\text { Ptje } \\
(126)\end{array}$ & $\begin{array}{c}\text { Escala } \\
(1-4)\end{array}$ & Nivel \\
\hline $\begin{array}{l}\text { Actividades en } \\
\text { clase }\end{array}$ & 816 & 3,00 & Alto & 365 & 2,90 & Medio \\
\hline Evaluación & 805 & 2,96 & Medio & 369 & 2,93 & Medio \\
\hline $\begin{array}{l}\text { Técnicas } \\
\text { didácticas }\end{array}$ & 788 & 2,90 & Medio & 355 & 2,82 & Medio \\
\hline $\begin{array}{l}\text { Medios instruc- } \\
\text { cionales }\end{array}$ & 586 & 2,16 & Medio & 271 & 2,15 & Medio \\
\hline $\begin{array}{l}\text { Participación de } \\
\text { los estudiantes }\end{array}$ & 819 & 3,01 & Alto & 369 & 2,93 & Medio \\
\hline Motivación & 769 & 2,83 & Medio & 348 & 2,76 & Medio \\
\hline Actitudes & 871 & 3,20 & Alto & 377 & 2,99 & Medio \\
\hline $\begin{array}{l}\text { Técnicas para } \\
\text { el conoc. y } \\
\text { habilidades }\end{array}$ & 789 & 2,90 & Medio & 357 & 2,83 & Medio \\
\hline \multicolumn{1}{|c|}{ Promedio } & 781 & 2,87 & Medio & 351 & 2,79 & Medio \\
\hline
\end{tabular}

Fuente: Encuesta realizada por Jesús Tello y Pedro Barrientos (30-10-2012).

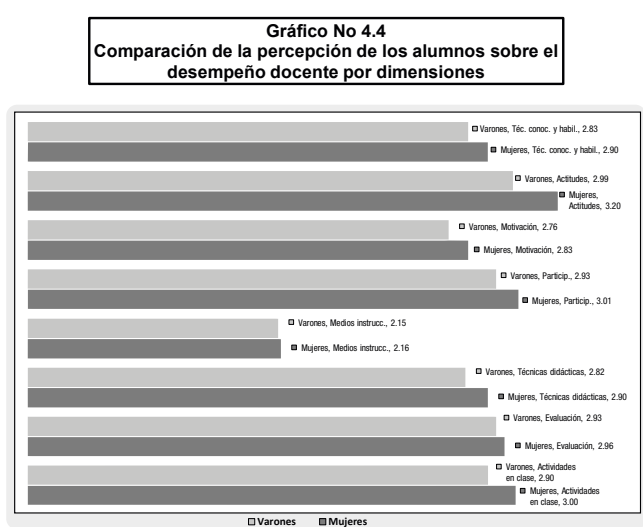

La tabla № 4.7, muestra que cualitativamente las alumnas y los alumnos tienen una percepción del desempeño docente en el nivel Medio en 7 dimensiones: Actividad en clase $(2,95)$, Evaluación $(2,94)$, Técnicas didácticas (2,86), Medios instruccionales $(2,16)$, Participación de los estudiantes (2,97), Motivación $(2,80)$ y Técnicas para el establecimiento de conocimientos y habilidades $(2,87)$, mientras que en la dimensión Actitudes el nivel de percepción de las alumnas y alumnos es Alto, ya que tiene un puntaje de 3,10 que los ubica en el tercio superior del recorrido de la escala

Tabla № 4.7: Comparación de la percepción de las alumnas y alumnos sobre el desempeño docente por dimensiones y total

\begin{tabular}{|l|c|c|c|c|c|c|}
\hline \multirow{2}{*}{ Dimensiones } & \multicolumn{2}{|c|}{ Mujeres } & \multicolumn{2}{c|}{ Varones } & \multicolumn{2}{c|}{ Total } \\
\cline { 2 - 7 } & $\begin{array}{c}\text { Escala } \\
(1-4)\end{array}$ & Nivel & $\begin{array}{c}\text { Escala } \\
(1-4)\end{array}$ & Nivel & $\begin{array}{c}\text { Escala } \\
(1-4)\end{array}$ & Nivel \\
\hline $\begin{array}{l}\text { Actividades en } \\
\text { clase }\end{array}$ & 3,00 & Alto & 2,90 & Medio & 2,95 & Medio \\
\hline Evaluación & 2,96 & Medio & 2,93 & Medio & 2,94 & Medio \\
\hline $\begin{array}{l}\text { Técnicas didác- } \\
\text { ticas }\end{array}$ & 2,90 & Medio & 2,82 & Medio & 2,86 & Medio \\
\hline $\begin{array}{l}\text { Medios instruc- } \\
\text { cionales }\end{array}$ & 2,16 & Medio & 2,15 & Medio & 2,16 & Medio \\
\hline $\begin{array}{l}\text { Participación de } \\
\text { los estudiantes }\end{array}$ & 3,01 & Alto & 2,93 & Medio & 2,97 & Medio \\
\hline Motivación & 2,83 & Medio & 2,76 & Medio & 2,80 & Medio \\
\hline Actitudes & 3,20 & Alto & 2,99 & Medio & 3,10 & Alto \\
\hline $\begin{array}{l}\text { Técnicas para el } \\
\text { conoc. y habili- } \\
\text { dades }\end{array}$ & 2,90 & Medio & 2,83 & Medio & 2,87 & Medio \\
\hline \multicolumn{1}{|c|}{ Promedio } & 2,87 & Medio & 2,79 & Medio & 2,83 & Medio \\
\hline
\end{tabular}

Fuente: Encuesta realizada por Jesús Tello y Pedro Barrientos (30-10-2012). 

presentan un mayor (781) puntaje acumulado en comparación al obtenido por los 126 alumnos (351). Comparando los índices porcentuales, se observa que las alumnas $(71,78 \%)$ presentan un índice mayor que el obtenido por los alumnos (69,64\%), además comparando los puntajes en la escala, se observa que también las alumnas $(2,87)$ presentan un puntaje mayor que los alumnos $(2,79)$, mientras que en la comparación cualitativa ambos grupos (alumnas y alumnos) presentan un nivel Medio en la escala. Estos resultados, nos permiten afirmar que las alumnas muestran una mejor percepción del Sistema de enseñanza-aprendizaje y evaluación aplicados por los profesores de la Región Junín.

Tabla No 4.8: Comparación de los puntajes de la percepción sobre la enseñanza-aprendizaje y evaluación

\begin{tabular}{|l|c|c|c|}
\hline \multicolumn{1}{|c|}{ Descripción } & $\begin{array}{c}\text { Mujeres } \\
(272)\end{array}$ & Relación & $\begin{array}{c}\text { Varones } \\
(126)\end{array}$ \\
\hline Puntaje acumulado & 781 & $>$ & 351 \\
\hline Índice porcentual & $71,78 \%$ & $>$ & $69,64 \%$ \\
\hline Puntaje en la escala (1-4) & 2,87 & $>$ & 2,79 \\
\hline Nivel cualitativo & Medio & $=$ & Medio \\
\hline
\end{tabular}

Fuente: Encuesta realizada por Jesús Tello y Pedro Barrientos (30-10-2012).

\section{PRUEBA DE LA HIPÓTESIS DE INVESTIGACIÓN Hipótesis específica 1}

La hipótesis específica 1 se formula de la siguiente manera:

Las mujeres poseen mejor nivel de percepción de la evaluación del desempeño docente de la Región Junín. Formulación de las hipótesis a contrastar:

$H_{0}$ : El nivel de percepción de la evaluación del desempeño docente no está asociado al género de los estudiantes.

$\mathrm{H}_{1}$ : El nivel de percepción de la evaluación del desempeño docente no está asociado al género de los estudiantes.

Para determinar el valor de la Chi cuadrada $\left(X_{c}^{2}\right)$ calculada fue necesario aplicar la siguiente fórmula:

Donde:

$\mathrm{X}_{\mathrm{c}}{ }_{\mathrm{c}}$ : Chi cuadrada calculada.

$0_{i j}$ : Percepción de las alumnas y alumnos sobre el desempeño docente.

$e_{i j}$ : Percepción esperada de las alumnas y alumnos sobre el desempeño docente.

En la tabla № 4.9 se muestra la tabla de contingencia para la prueba de hipótesis, donde se ubica los valores esperados y los valores observados respectivamente:

Tabla № 4.9: Tabla de contingencia de los niveles de percepción del desempeño docente por género

\begin{tabular}{|c|c|c|c|c|}
\hline Niveles & Bajo & Medio & Alto & Total \\
\hline Mujeres & & & & 8 \\
\hline Varones & & & & 8 \\
\hline Total & 0 & 13 & 3 & 16 \\
\hline
\end{tabular}

El valor teórico de la prueba Chi cuadrada $\left(X^{2}{ }_{a}\right)$ para un nivel de significancia $\alpha=0,05$ y grados de libertad: $g . l=$ $(3-1)(2-1)=2 \times 1=2$ es: $X_{\alpha}^{2}=5,991$

El valor de la Chi cuadrada calculada: $X^{2}{ }_{c}=3,602$ Regla de decisión: Se rechaza $\mathrm{H}_{0}$ si ocurre que:

$$
X^{2}{ }_{c}>X^{2}{ }_{\alpha}
$$

$$
3,602>5,991
$$

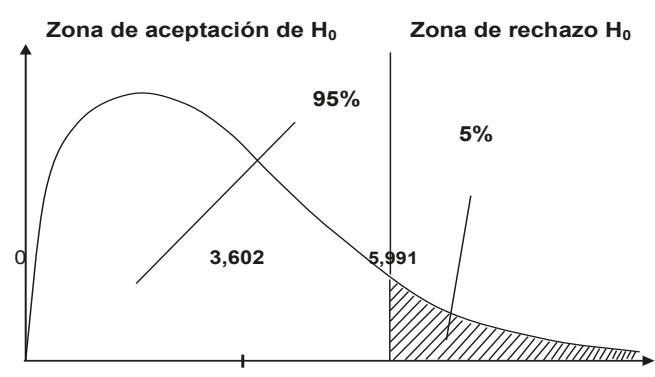


Como la relación es Falsa, entonces con un nivel de significación de $\alpha=0,05$ y para 2 grados de libertad no es posible rechazar la hipótesis nula $\left(\mathrm{H}_{0}\right)$, por lo tanto se acepta: El nivel de percepción de la evaluación del desempeño docente no está asociado al género de los estudiantes.

Al demostrarse la validez de la hipótesis nula se demuestra que: Las mujeres no poseen mejor nivel de percepción de la evaluación del desempeño docente de la Región Junín.

\section{HIPÓTESIS GENERAL}

La hipótesis general de investigación se formula de la siguiente manera:

Existe un nivel medio de percepción de la evaluación del desempeño docente en la Región Junín.

Formulación de las hipótesis a contrastar:

$\mathrm{H}_{0}$ : No hay diferencia en las frecuencias de los niveles de percepción de la evaluación del desempeño docente.

$\mathrm{H}_{1}$ : Hay diferencia en las frecuencias de los niveles de percepción de la evaluación del desempeño docente.

En la tabla № 4.10 se muestra la tabla de contingencia para la prueba de hipótesis:

Tabla № 4.10

Tabla de contingencia de los niveles de percepción del desempeño docente

\begin{tabular}{|c|c|c|c|c|}
\hline Niveles & Bajo & Medio & Alto & Total \\
\hline Frecuencias & & & & 8 \\
\hline
\end{tabular}

El valor teórico de la prueba Chi cuadrada $\left(X^{2}{ }_{\alpha}\right)$ para un nivel de significancia $\alpha=0,05$ y grados de libertad: $g . \mathrm{l} .=$ $3-1=2$ es:

$$
X_{\alpha}^{2}=5,991
$$

El valor de la Chi cuadrada calculada: $\quad X^{2}{ }_{c}=10,737$ Regla de decisión: Se rechaza $\mathrm{H}_{0}$ si ocurre que:

$$
X^{2}{ }_{c}>X^{2}{ }_{\alpha}
$$

$$
10,737>5,991
$$

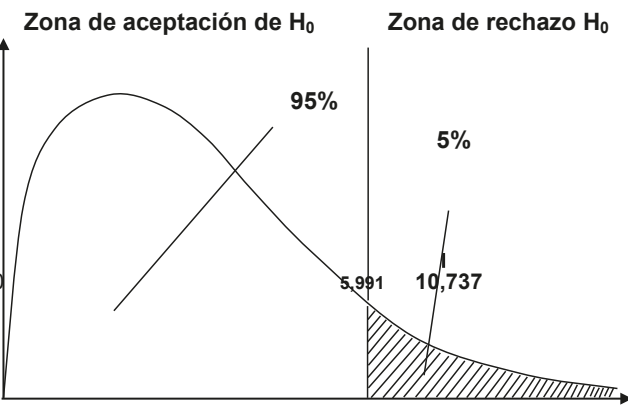

Como la relación es Verdadera, entonces con un nivel de significación de $\mathbf{\alpha}=0,05$ y para 2 grados de libertad se rechaza la hipótesis nula $\left(\mathrm{H}_{0}\right)$ y se acepta la hipótesis alterna $H_{1}$, por lo tanto se acepta que: Hay diferencia en las frecuencias de los niveles de percepción de la evaluación del desempeño docente.

Como se observa la mayor frecuencia en el nivel Medio, y se ha demostrado que existe diferencias en las frecuencias, entonces se demuestra la hipótesis de investigación: Existe un nivel medio de percepción de la evaluación del desempeño docente en la Región Junín.

\section{DISCUSIÓN}

De los resultados del trabajo de investigación, podemos manifestar que los docentes de la Región Junín están en un nivel medio de desempeño profesional, las percepciones de los estudiantes de ambos géneros en relación al desenvolvimiento de sus profesores es preocupante, la educación es un elemento o factor importante en el desarrollo cultural, económico y social de las regiones y del país.

Pero las opiniones de los estudiantes en relación a la enseñanza que ejecutan sus profesores en las diferentes materias del currículo de secundaria es preocupante. Lo óptimo sería que los profesores demuestre ante los estudiantes un resultado óptimo en su desempeño profesional.

\section{CONCLUSIONES}

1. Con un nivel de significación $\boldsymbol{\alpha}=0,05$ y para 2 grados de libertad se concluye que: El nivel de percepción de la evaluación del desempeño docente no está asociado al género de los estudiantes.

2. Se demuestra estadísticamente que las mujeres no 
poseen mejor nivel de percepción de la evaluación del desempeño docente de la Región Junín, afirmación que se hace para un 95\% de nivel de confianza y $5 \%$ de nivel de significación.

3. Existe diferencia en las frecuencias de los niveles de percepción de la evaluación del desempeño docente, para un nivel de significación $\mathbf{\alpha}=0,05$ y para 2 grados de libertad.

4. Se demuestra mediante la estadística inferencial que existe un nivel Medio de percepción de la evaluación del desempeño docente en la Región Junín, conclusión que se hace para un 95\% de nivel de confianza y $5 \%$ de nivel de significación y 2 grados de libertad.

5. Las alumnas $(2,87)$ presentan un puntaje mayor que los alumnos $(2,79)$ en la escala 1-4 de percepción del desempeño docente de la región Junín, lo que cualitativamente equivale a un nivel un nivel Medio en la escala en ambos grupos, esta afirmación se hace en base al contenido de la tabla № 4.8.

6. Cualitativamente las alumnas y los alumnos tienen una percepción del desempeño docente en el nivel Medio en 7 dimensiones: Actividad en clase $(2,95)$, Evaluación (2,94), Técnicas didácticas $(2,86)$, Medios instruccionales $(2,16)$, Participación de los estudiantes $(2,97)$, Motivación $(2,80)$ y Técnicas para el establecimiento de conocimientos y habilidades $(2,87)$, mientras que en la dimensión Actitudes el nivel de percepción de las alumnas y alumnos es Alto, ya que tiene un puntaje de 3,10, afirmación que se hace en base a la tabla № 4.7 .

\section{REFERENCIAS BIBLIOGRÁFICAS}

- BARRIENTOS G. Pedro (2005).Investigación Científica. Tarea cotidiana del docente. UGRAPH S.A.C. Lima-Perú.

- BERNARD, Juan Antonio (2000). Modelo cognitivo de evaluación educativa, Madrid - España: Ediciones NARCEA S.A.

- Bologna, E. (2011). Estadística para psicología y Educación. Argentina: Brujas.

- Christensen, Howard. (1994). Estadística paso a paso. México. Trillas.

- CERDA GUTIÉRREZ, Hugo (2000). La evaluación como experiencia total. Logros-objetivos-procesos-competencias y desempeño, Santa Fe de Bogo- tá- Colombia: Cooperativa Editorial Magisterio.

- DIAZ BARRIGA, Frida y HERNÁNDEZ, Gerardo (2002). Estrategias docentes para un aprendizaje significativo: una interpretación constructivista, 2da. Ed., México: Mc Graw-Hill.

- Fernández, M. y otros (2009). Curso básico de psicometría. Lugar editorial. Buenos Aires.

- FLOREZ OCHOA, Rafael (2001). Evaluación pedagógica y cognición, 3ra. Ed., Santa Fe de Bogotá: Editorial Mc Graw-Hill Interamericana S. A.

- JIMENEZ PASNIAGUA, LUIS; MARCOS BARRIO, JOSÉ; FUENTES IGLESIAS, RUBEN (2010). Aprendizaje interactivo, Universidad Complutense de Madrid.

- Lind, D. y otros. (2004) Estadística para administración y economía. Bogotá.

- Kaplan, R. y Sacuzzo, D. (2006) Pruebas psicológicas. Sexta edición. México.

- Martín, Quintín y otros. (2008). Tratamiento estadístico de datos con SPSS. Madrid. Thomson.

- MINISTERIO DE EDUCACIÓN (2002). Manual para Docentes de Educación Primaria, UCAD - DINFOCAD Lima - Perú.

- MINISTERIO DE EDUCACIÓN (2007). Guía de Evaluación del Aprendizaje, Lima - Perú: QUEBECOR WORLD PERU S.A., p. 6-15, 25-40.

- MINISTERIO DE EDUCACIÓN (2008). Diseño Curricular Nacional, Lima - Perú.

- OVALLE CARRANZA, DEMETRIO ARTURO Y JIMÉNEZ BUILES, JOVANI ALBERTO(S/F). Sistemas de Enseñanza / Aprendizaje basados en Agentes Inteligentes Pedagógicos,

- UNIVERSIDAD NACIONAL DE COLOMBIA. Escuela de Sistemas.

- Quezada, Nel. (2009). Estadísticas con PASW 18. Lima: Empresa editora Macro EIRL.

- SALINAS, Dino (2002). ¡Mañana examen! La evaluación entre la teoría y la realidad, Barcelona '- España: GRAÓ, p. 7-108, [disponible en: http:// books.google.com/books?id=].

- Tello Yance Jesús (2013). Metodología de investigación científica y educativa, Ediciones Grapex Perú SRL. - Triola, M. (2010). Estadística. Décima edición. México: Pearson Educación. 